\title{
Another product construction for large sets of resolvable directed triple systems
}

\author{
Hongtao Zhao* \\ School of Mathematics and Physics \\ North China Electric Power University, Beijing 102206, China \\ ht_zhao@163.com \\ Submitted: Jul 26, 2009; Accepted: Sep 13, 2009; Published: Sep 18, 2009 \\ Mathematics Subject Classifications: 05B07
}

\begin{abstract}
A large set of resolvable directed triple systems of order $v$, denoted by LRDTS $(v)$, is a collection of $3(v-2) \operatorname{RDTS}(v) \mathrm{s}$ based on $v$-set $X$, such that every transitive triple of $X$ occurs as a block in exactly one of the $3(v-2) \operatorname{RDTS}(v)$ s. In this paper, we use DTRIQ and LR-design to present a new product construction for $\operatorname{LRDTS}(v) \mathrm{s}$. This provides some new infinite families of $\operatorname{LRDTS}(v) \mathrm{s}$.
\end{abstract}

\section{Introduction}

Let $X$ be a $v$-set. In what follows, an ordered pair of $X$ is always an ordered pair $(x, y)$, where $x \neq y \in X$. A transitive triple on $X$ is a set of three ordered pairs $(x, y)$, $(y, z)$ and $(x, z)$ of $X$, which is denoted by $(x, y, z)$.

A directed triple system of order $v$, denoted by $\operatorname{DTS}(v)$, is a pair $(X, \mathcal{B})$ where $\mathcal{B}$ is a collection of transitive triples on $X$, called blocks, such that each ordered pair of $X$ occurs in exactly one block of $\mathcal{B}$. A DTS $(v)$ is called resolvable and is denoted by $\operatorname{RDTS}(v)$ if its blocks can be partitioned into subsets (called parallel classes), each containing every element of $X$ exactly once.

A large set of directed triple systems of order $v$, denoted by $\operatorname{LDTS}(v)$, is a collection of $3(v-2)$ DTS $(v)$ s based on $X$ such that every transitive triple from $X$ occurs as a block in exactly one of the $3(v-2)$ DTS $(v)$ s. Existence results for LDTSs and RDTSs are well known from $[1,9]$.

Theorem $1.1(1)$ There exists an $\operatorname{LDTS}(v)$ if and only if $v \equiv 0,1(\bmod 3)$ and $v \geqslant 3$.

(2) There exists an $\operatorname{RDTS}(v)$ if and only if $v \equiv 0(\bmod 3), v \geqslant 3$ and $v \neq 6$.

\footnotetext{
${ }^{*}$ Research supported by NSFC Grant 10901051, NSFC Grant 10971051 and Doctoral Grant of North China Electric Power University.
} 
A large set of disjoint $\operatorname{RDTS}(v) \mathrm{s}$ is denoted by LRDTS $(v)$. The existence of LRDTS $(v) \mathrm{s}$ has been investigated by Kang [8], Kang and Lei [10], Kang and Tian [11], Kang and Xu [12], Kang and Zhao [13], Xu and Kang [17] and Zhou and Chang [22, 23]. By their research and related results about large sets of Kirkman triple systems [3, 4, 5, 6, 14, 15, 18, 19, 20, 21], we can list the known results as follows.

Theorem 1.2 There exists an LRDTS $(v)$ for the following orders $v$ :

(1) $v=3^{k} m$, where $k \geqslant 1$ and $m \in\{1,4,5,7,11,13,17,23,25,35,37,41,43,47,53,55$, $57,61,65,67,91,123\} \cup\left\{2^{2 r+1} 25^{s}+1: r \geqslant 0, s \geqslant 0\right\}$.

(2) $v=7^{k}+2,13^{k}+2,25^{k}+2,2^{4 k}+2$ and $2^{6 k}+2$, where $k \geqslant 0$.

(3) $v=12(t+1)$, where $t \in\{0,1,2,3,4,6,7,8,9,14,16,18,20,22,24\}$.

(4) $v=6 t+3$, where $t \in\{35,38,46,47,48,51,56,60\}$.

(5) $v=\left(3 \prod_{i=1}^{p}\left(2 q_{i}^{r_{i}}+1\right) \prod_{j=1}^{q}\left(4^{s_{j}}-1\right)\right)$, where $p+q \geqslant 1, r_{i}, s_{j} \geqslant 1$ and prime power $q_{i} \equiv 7(\bmod 12)$.

Also, if there exists an $\operatorname{LRDTS}(v)$, then there exists an $\operatorname{LRDTS}\left(\left(2 \cdot s^{k}+1\right) v\right)$ for any $k \geqslant 0, s=7,13$ and $v \equiv 0,3,9(\bmod 12)$.

A group-divisible design (briefly $\mathrm{GDD})$ is a triple $(X, \mathcal{G}, \mathcal{B})$ with the following properties: (i) $X$ is a finite set of points; (ii) $\mathcal{G}$ is a partition of $X$ into subsets called groups; (iii) $\mathcal{B}$ is a set of subsets of $X$ (called blocks) such that a group and a block contain at most one common point, and any pair of points from distinct groups occur in exactly one block of $\mathcal{B}$. A $\operatorname{GDD}(X, \mathcal{G}, \mathcal{B})$ is called resolvable, denoted by RGDD, if there exists a partition $\Gamma=\left\{P_{1}, P_{2}, \cdots, P_{r}\right\}$ of $\mathcal{B}$ such that each part $P_{i}$ (called parallel classes) is a partition of $X$.

A GDD is called a transversal design if it has exactly $k$ groups of size $n$ and every block has size $k$. We denoted such a GDD by $\operatorname{TD}(k, n)$. A TD is called resolvable (denoted by RTD) if it is a RGDD.

A $\operatorname{GDD}(X, \mathcal{G}, \mathcal{B})$ is called a Steiner triple system if $|X|=v$ and it has $v$ groups of size 1 and every block has size 3 . Such a GDD is denoted briefly by $\operatorname{STS}(v)(X, \mathcal{B})$. A resolvable $\operatorname{STS}(v)$ is called a Kirkman triple system and denoted by $\operatorname{KTS}(v)$.

A large set of Kirkman triple system of order $v$, denoted by $\operatorname{LKTS}(v)$, is a collection of $v-2 \operatorname{KTS}(v)$ s based on a $v$-set $X$, such that each triple from $X$ occurs in exactly one of the $v-2 \operatorname{KTS}(v) \mathrm{s}$. In a $\operatorname{KTS}(v)$, if we replace any triple $\{x, y, z\}$ by three collections of transitive triples $\{(x, y, z),(z, y, x)\},\{(y, z, x),(x, z, y)\}$ and $\{(z, x, y),(y, x, z)\}$, then we obtain three $\operatorname{RDTS}(v)$ s. It is obvious that the existence of an $\operatorname{LKTS}(v)$ implies the existence of an $\operatorname{LRDTS}(v)$. However, this approach can provide only odd orders of $v$ since the existence of a $\operatorname{KTS}(v)$ implies $v \equiv 3(\bmod 6)$. The existence of $\operatorname{LKTS}(v) \mathrm{s}$, known as the general Sylvester's problem of the 15 schoolgirls, has a long history [3]. Some orders in Theorem 1.2 come from the existence of $\operatorname{LKTS}(v) \mathrm{s}$.

The main result of this paper is to give a new product construction for LRDTSs. This provides some new infinite families of $\operatorname{LRDTS}(v)$ s. In Section 2, we give some concepts such as $\operatorname{TRIQ}(v), \operatorname{DTRIQ}(v)$ and $\operatorname{LR}(u)$, etc. In Section 3, we make use of $\operatorname{DTRIQ}(v)$ and $\operatorname{LR}(u)$ to present a new product construction. In Section 4, we give new orders for $\operatorname{LRDTS}(v) \mathrm{s}$. 


\section{Definitions}

A quasigroup is a pair $(X, \circ)$, where $X$ is a set and (o) is a binary operation on $X$ such that the equation $a \circ x=b$ and $y \circ a=b$ are uniquely solvable for every pair of elements $a, b$ in $X$. The order of a quasigroup $(X, \circ)$ is the size of $X$.

A quasigroup of order $v$ is called idempotent if the identity $x \circ x=x$ holds for all $x$ in $X$. An idempotent quasigroup of order $v$ is denoted by $\operatorname{IQ}(v)$. A quasigroup of order $v$ is called symmetric if the identity $x \circ y=y \circ x$ holds for every pair of elements $x, y$ in $X$. An symmetric quasigroup of order $v$ is denoted by $\mathrm{SQ}(v)$.

A quasigroup $(X, \circ)$ is called resolvable if all $v(v-1)$ pairs of distinct elements can be partitioned into subsets $T_{i}, 1 \leqslant i \leqslant 3(v-1)$, such that every $\left\{(x, y, x \circ y):(x, y) \in T_{i}\right\}$ is a partition of $X$. An idempotent quasigroup $\mathrm{IQ}(v)$ is called first transitive if there exists a group of order $v$ acting transitively on $X$ which forms an automorphism group of the $\operatorname{IQ}(v)$. A first transitive resolvable $\operatorname{IQ}(v)$ is denoted by $\operatorname{TRIQ}(v)$. A first transitive resolvable symmetric $\operatorname{IQ}(v)$ is denoted by $\operatorname{TRISQ}(v)$.

For an idempotent quasigroup $(Y, \circ)$ and for each ordered pair $(i, j), i \neq j \in\{0,1,2\}$, define a collection of transitive triples from $\{i, j\} \times Y$ as follows.

$$
\begin{aligned}
& T(i, j)=\bigcup_{\substack{x \neq y \in Y\\
}} t(x, y, x \circ y), \text { where } \\
& \quad t(x, y, x \circ y)=\{((i, x),(i, y),(j, x \circ y)),((i, x),(j, x \circ y),(i, y)),((j, x \circ y),(i, x),(i, y))\} .
\end{aligned}
$$

An idempotent quasigroup $(Y, \circ)$ is called second transitive provided that $T(i, j)$ can be partitioned into three sets $T_{0}(i, j), T_{1}(i, j)$ and $T_{2}(i, j)$ such that

i) the three transitive triples in $t(x, y, x \circ y)$ belong to $T_{0}, T_{1}$ and $T_{2}$, respectively;

ii) if $a \neq b \in Y$, each of the ordered pairs $((i, a),(j, b))$ and $((j, b),(i, a))$ belongs to exactly one transitive triple in each of $T_{0}(i, j), T_{1}(i, j)$ and $T_{2}(i, j)$.

An IQ $(v)$ with both first and second transitivity is called doubly transitive and is denoted by DTRIQ $(v)$. In [22], Zhou and Chang gave the following existence result.

Lemma 2.1 There exists a $\operatorname{DTRIQ}(v)$ for any positive integer $v \equiv 0,3,9(\bmod 12)$.

Transitive IQ has been used to give a tripling construction for large sets of STSs in Teirlinck [16]. To consider the similar problem for large sets of KTSs and large sets of RDTSs, we demand that the transitive IQ must have certain property of resolvability. TRISQ $(v)$ was used to construct LKTSs [20]. DTRIQ $(v)$ was used to construct LRDTSs $[22,23]$.

In [14], Lei introduce a kind of combinatorial design named LR-design, denoted by $\operatorname{LR}(u)$. An $\operatorname{LR}(u)$ is a collection $\left\{\left(X, \mathcal{A}_{k}^{j}\right): 1 \leqslant k \leqslant \frac{u-1}{2}, j=0,1\right\}$, where each $\left(X, \mathcal{A}_{k}^{j}\right)$ is a $\operatorname{KTS}(u)$ based on $u$-set $X$ and $\left\{A_{k}^{j}(h) ; 1 \leqslant h \leqslant \frac{u-1}{2}\right\}$ is a resolution (collection of parallel classes) of $\mathcal{A}_{k}^{j}$ with the properties.
i) $\bigcup_{k=1}^{\frac{u-1}{2}} A_{k}^{0}(1)=\bigcup_{k=1}^{\frac{u-1}{2}} A_{k}^{1}(1)=\mathcal{A}$ forms a $\operatorname{KTS}(u)$ over $X$ too;
ii) Any triple from $X$ is contained in $\bigcup_{k=1}^{\frac{u-1}{2}} \bigcup_{j=0}^{1} \mathcal{A}_{k}^{j}$.

Lei [14] and Ji and Lei [7] obtained some existence results for $\operatorname{LR}(u)$. 
Lemma $2.2[14,7]$ There exists an $\operatorname{LR}(u)$ for $u=3^{n}, 2 \cdot 13^{n}+1$ and $2 \cdot 7^{n}+1$, where $n \geqslant 1$.

Recently, using these auxiliary designs and their existence, Chang et al. [22, 23] proved the following conclusions.

Lemma 2.3 [22] If there exist both a DTRIQ $(v)$ and an $\operatorname{LRDTS}(v)$, then there exists an $\operatorname{LRDTS}(3 v)$.

Lemma 2.4 [23] If there exist an $\operatorname{LRDTS}(v), a \operatorname{DTRIQ}(v)$ and an $\operatorname{LR}(u)$, then there exists an $\operatorname{LRDTS}(u v)$.

Next, we introduce the concept of complete mapping in a finite group. We follow the definition in Denes and Keedwell [2].

A complete mapping of a group $(G, \cdot)$, is a bijection mapping $x \rightarrow \theta(x)$ of $G$ upon $G$, such that the mapping $\eta(x)=x \cdot \theta(x)$ is also a bijection mapping of $G$ upon $G$. The following existence results were stated in [2].

Lemma 2.5 [2] If $G$ is an arbitrary group of order $n=4 k+2$, then $G$ has no complete mapping. If $G$ is an abelian group of order $n \neq 4 k+2$, then $G$ does have a complete mapping.

Let $X=\{0,1, \cdots, v-1\}$ and $(X, \circ)$ be an idempotent quasigroup with a sharply transitive automorphism group $G$ written multiplicatively. It is easy to see that there is a unique $g \in G$ such that $g(x)=y$ for every pair of elements $x, y$ in $X$. Let the first row of $(X, \circ)$ be of the following ordered triples:

$$
\left(0, h(0), h^{*}(0)\right), h \in G .
$$

Then $h \mapsto h^{*}$ is a bijection between $G$, denoted by $\Phi$. Hence, $\left(g(0), g h(0), g h^{*}(0)\right), g, h \in G$ forms the quasigroup $(X, \circ)$.

Then $\left(g, g h, g h^{*}\right), g, h \in G$ is a latin square on $G$, which implies that

$$
\left\{\left(g h, g h^{*}\right): g, h \in G\right\}=G \times G .
$$

So, we have

$$
\left\{h\left(h^{*}\right)^{-1}: h \in G\right\}=G
$$

Note that the mapping $\bar{\Phi}: h \mapsto\left(h^{*}\right)^{-1}$ is also a bijection between $G$. By the definition of complete mapping and formula (1), $\bar{\Phi}$ is a complete mapping of $G$. Next we record the result as follows.

Lemma 2.6 If there exists a transitive IQ with $G$ as a sharply transitive automorphism group, then $G$ has a complete mapping. 


\section{A new product construction for LRDTS}

Let $X=\{0,1, \cdots, v-1\}$ and $(X, \circ)$ be an idempotent quasigroup with a sharply transitive automorphism group $G=\left\{\sigma_{0}, \sigma_{1}, \cdots, \sigma_{v-1}\right\}$. By Lemma 2.6, $G$ has a complete mapping, say, $\Phi^{-1}$. Let $\sigma^{*}=\Phi(\sigma)$ for $\sigma \in G$. Then, by the definition of complete mapping, we have

$$
\left\{\sigma\left(\sigma^{*}\right)^{-1}: \sigma \in G\right\}=G
$$

Theorem 3.1 If there exist an $\operatorname{LRDTS}(3 v), a$ DTRIQ $(v)$ and an $\operatorname{LR}(u)$, then there exists an $\operatorname{LRDTS}(u v)$.

Proof. Suppose that $X$ is a set of size $u$ with a linear order " $<$ " (i.e. for any $x \neq y$, $x, y \in X$, either $x<y$ or $y<x)$. We have an $\operatorname{LR}(u)$ over $X$ with the following collection of $u-1 \operatorname{KTS}(u)$

$$
\left\{\left(X, \mathcal{A}_{k}^{l}\right): 1 \leqslant k \leqslant \frac{u-1}{2}, l=0,1\right\}
$$

which with following properties:

(i) Let the resolution of $\mathcal{A}_{k}^{l}$ be $\Gamma_{k}^{l}=\left\{A_{k}^{l}(h): 1 \leqslant h \leqslant \frac{u-1}{2}\right\}$, and

$$
\bigcup_{k=1}^{\frac{u-1}{2}} A_{k}^{0}(1)=\bigcup_{k=1}^{\frac{u-1}{2}} A_{k}^{1}(1)=\mathcal{A},
$$

$(X, \mathcal{A})$ is a $\operatorname{KTS}(u)$.

(ii) For any triple $T=\{x, y, z\} \subset X, x \neq y \neq z \neq x$, there exist $k, l$ such that $T \in \mathcal{A}_{k}^{l}$.

Furthermore, suppose that $Y$ is a set of size $v$. So we have a DTRIQ $(v)$ over $Y$. Let $(Y, \circ)$ be a $\operatorname{DTRIQ}(v), G=\left\{\sigma_{0}, \sigma_{1}, \cdots, \sigma_{v-1}\right\}$ be the transitive automorphism group of $(Y, \circ)$. We will construct an $\operatorname{LRDTS}(u v)$ on the point set $X \times Y$. The construction proceeds in 2 steps.

Step 1: For any $\{x, y, z\} \subseteq X,\{x, y, z\} \in \mathcal{A}=\bigcup_{k=1}^{\frac{u-1}{2}} A_{k}^{0}(1)$.

(1). If $\{x, y, z\} \in A_{1}^{0}(1)$, we have an $\operatorname{LRDTS}(3 v)$ on the point set $\{x, y, z\} \times Y$. Let its block set be $\left\{\mathcal{B}_{i, m}^{\{x, y, z\}}: 1 \leqslant i \leqslant v-2, m=0,1,2\right\} \bigcup\left\{\mathcal{B}_{j, m}^{l}(\{x, y, z\}): 0 \leqslant j \leqslant\right.$ $v-1, l=0,1, m=0,1,2\}$, and each $\mathcal{B}_{i, m}^{\{x, y, z\}}$ can be partitioned into parallel classes $B_{i, m}^{\{x, y, z\}}(n), 1 \leqslant n \leqslant 3 v-1$, each $\mathcal{B}_{j, m}^{l}(\{x, y, z\})$ can be partitioned into parallel classes $B_{j, m}^{l}(\{x, y, z\}, n), 1 \leqslant n \leqslant 3 v-1$.

(2). If $\{x, y, z\} \notin A_{1}^{0}(1)$, i.e. $\{x, y, z\} \in A_{k}^{0}(1)$ for some $k, 2 \leqslant k \leqslant \frac{u-1}{2}, x<y<z$, let

$$
\begin{aligned}
P_{j, s}^{\{x, y, z\}} & =\left\{(x, a),\left(y, \sigma_{s}(a)\right),\left(z, \sigma_{j} \sigma_{s}^{*}(a)\right): a \in Y\right\}, \\
P_{0, j, s}^{\{x, y, z\}} & =\left\{(u, v, w),(w, v, u):\{u, v, w\} \in P_{j, s}^{\{x, y, z\}}\right\}, \\
P_{1, j, s}^{\{x, y\}} & =\left\{(u, w, v),(v, w, u):\{u, v, w\} \in P_{j, s}^{\{x, y, z\}}\right\}, \\
P_{2, j, s}^{\{x, y, z\}} & =\left\{(w, u, v),(v, u, w):\{u, v, w\} \in P_{j, s}^{\{x, y, z\}}\right\},
\end{aligned}
$$


where $\sigma_{s}, \sigma_{j} \in G$ and let

$$
\mathcal{A}_{m, j}^{\{x, y, z\}}=\bigcup_{\sigma_{s} \in G} P_{m, j, s}^{\{x, y, z\}}, m=0,1,2 .
$$

So we have: (1) For $x^{\prime} \neq y^{\prime} \in\{x, y, z\}, a, b \in Y$, each of the order pair $((x, a),(y, b))$ and $((y, b),(x, a))$ belongs to exactly one triple of $\mathcal{A}_{m, j}^{\{x, y, z\}} ;(2) \mathcal{A}_{m, j}^{\{x, y, z\}}$ and $\mathcal{A}_{m^{\prime}, j^{\prime}}^{\{x, y, z}$ are disjoint for $(m, j) \neq\left(m^{\prime}, j^{\prime}\right)$.

Since $(Y, \circ)$ is a $\operatorname{DTRIQ}(v)$, for any ordered pair $(a, b) \in Y \times Y(a \neq b)$ and any $\sigma \in G$, we get an element $a \circ b$ in $Y$ such that $\sigma(a) \circ \sigma(b)=\sigma(a \circ b)$. For $0 \leqslant j \leqslant v-1$, define six permutations on $Y$, namely $\alpha_{j}^{(s)}, \beta_{j}^{(s)}\left(s \in Z_{3}\right)$ as follows:

$$
\begin{gathered}
\alpha_{j}^{(0)}=\sigma_{j}, \quad \alpha_{j}^{(1)}=\sigma_{0} \sigma_{j}^{*} \sigma_{j}^{-1}, \quad \alpha_{j}^{(2)}=\left(\sigma_{0} \sigma_{j}^{*}\right)^{-1}=\left(\alpha_{j}^{(1)} \alpha_{j}^{(0)}\right)^{-1}, \\
\beta_{j}^{(0)}=\sigma_{v-1} \sigma_{j}^{*}, \quad \beta_{j}^{(1)}=\sigma_{j}\left(\sigma_{v-1} \sigma_{j}^{*}\right)^{-1}, \quad \beta_{j}^{(2)}=\sigma_{j}^{-1}=\left(\beta_{j}^{(1)} \beta_{j}^{(0)}\right)^{-1} .
\end{gathered}
$$

Here, if $\pi$ is a permutation of $Y$, we denote by $\pi T_{m}(u, v)$ the transitive triples obtained by replacing each occurrence of $(u, a)$ with $(u, \pi(a))$ (and keeping those occurrences with the first component " $u$ " unchanged). Using the six permutations defined above, for each $m \in\{0,1,2\}$ and $j \in\{0,1, \cdots, v-1\}$, define

$$
\begin{aligned}
& \mathcal{C}_{j, m}^{0}=\alpha_{j}^{(0)} T_{m}(x, y) \cup \alpha_{j}^{(1)} T_{m}(y, z) \cup \alpha_{j}^{(2)} T_{m}(z, x), \\
& \mathcal{C}_{j, m}^{1}=\beta_{j}^{(0)} T_{m}(x, y) \cup \beta_{j}^{(1)} T_{m}(y, z) \cup \beta_{j}^{(2)} T_{m}(z, x),
\end{aligned}
$$

and

$$
\mathcal{B}_{j, m}^{l}(\{x, y, z\})=P_{m, v-l, j}^{\{x, y\}} \bigcup \mathcal{C}_{j, m}^{l},
$$

where $0 \leqslant j \leqslant v-1, m=0,1,2, l=0,1$ and $v-l=0, v-1$.

Furthermore, $\left(\{x, y, z\} \times Y, \mathcal{B}_{j}^{l}(\{x, y, z\})\right), 0 \leqslant j \leqslant v-1, l=0,1$, is an $\operatorname{RDTS}(3 v)$. Let each $\mathcal{B}_{j, m}^{l}(\{x, y, z\})$ can be partitioned into parallel classes $B_{j, m}^{l}(\{x, y, z\}, n), 1 \leqslant n \leqslant$ $3 v-1$.

(For any triple $T$ of $X \times Y, T$ is form as $((x, a),(x, b),(x, c))$ or $((x, a),(x, b),(y, c))$ or $((x, a),(y, b),(x, c))$ or $((y, a),(x, b),(x, c))$ or $((x, a),(y, b),(z, c))$ with $\{x, y, z\} \in \mathcal{A}$, then $T$ appears in Step 1.)

Step 2: For any $\{x, y, z\} \subseteq X, x<y<z,\{x, y, z\} \notin \mathcal{A}$, (i.e. there exist $k, l$ such that $\left.\{x, y, z\} \in \mathcal{A}_{k}^{l} \backslash A_{k}^{l}(1)\right)$ define $\mathcal{A}_{m, j}^{\{x, y, z\}}$ like Step 1 .

Define

$$
\mathcal{C}_{m, i}=\left(\bigcup_{\{x, y, z\} \in \mathcal{A} \backslash A_{1}^{0}(1)} \mathcal{A}_{m, i}^{\{x, y, z\}}\right) \bigcup\left(\bigcup_{\{x, y, z\} \in A_{1}^{0}(1)} \mathcal{B}_{i, m}^{\{x, y, z\}}\right) .
$$

It is not difficult to check that each $\left(X \times Y, \mathcal{C}_{m, i}\right), 1 \leqslant i \leqslant v-2, m=0,1,2$, is an $\operatorname{RDTS}(u v)$ with the following parallel classes:

$$
C_{m, i}(n)=\bigcup_{\{x, y, z\} \in A_{1}^{0}(1)} B_{i, m}^{\{x, y, z\}}(n), 1 \leqslant n \leqslant 3 v-1 ;
$$




$$
\begin{aligned}
& C_{m, i}(k, s)=\bigcup_{\{x, y, z\} \in A_{k}^{0}(1)}\left\{(u, v, w):\{u, v, w\} \in P_{m, i, s}^{\{x, y, z\}}\right\}, 2 \leqslant k \leqslant \frac{u-1}{2}, 0 \leqslant s \leqslant v-1 . \\
& \bar{C}_{m, i}(k, s)=\bigcup_{\{x, y, z\} \in A_{k}^{0}(1)}\left\{(w, v, u):\{u, v, w\} \in P_{m, i, s}^{\{x, y, z\}}\right\}, 2 \leqslant k \leqslant \frac{u-1}{2}, 0 \leqslant s \leqslant v-1 .
\end{aligned}
$$

Furthermore, these $3(v-2)$ RDTSs are obviously disjoint.

Define

$$
\mathcal{D}_{m, k, j}^{l}=\left(\bigcup_{\{x, y, z\} \in A_{k}^{l}(1)} \mathcal{B}_{j, m}^{l}(\{x, y, z\})\right) \bigcup\left(\bigcup_{\{x, y, z\} \in \mathcal{A}_{k}^{l} \backslash A_{k}^{l}(1)} \mathcal{A}_{m, j}^{\{x, y, z\}}\right),
$$

where $1 \leqslant k \leqslant \frac{u-1}{2}, 0 \leqslant j \leqslant v-1, m=0,1,2, l=0,1$. It is not difficult to check that each $\left(X \times Y, \mathcal{D}_{m, k, j}^{l}\right)$ is an $\operatorname{RDTS}(u v)$ with the following parallel classes:

$$
\begin{gathered}
\mathcal{D}_{m, k, j}^{l}(n)=\bigcup_{\{x, y, z\} \in A_{k}^{l}(1)} \mathcal{B}_{j, m}^{l}(\{x, y, z\}, n), 1 \leqslant n \leqslant 3 v-1, \\
\mathcal{D}_{m, k, j}^{l}(h, s)=\bigcup_{\{x, y, z\} \in A_{k}^{l}(h)}\left\{(u, v, w):\{u, v, w\} \in P_{m, j, s}^{\{x, y, z\}}\right\}, 2 \leqslant h \leqslant \frac{u-1}{2}, 0 \leqslant s \leqslant v-1 . \\
\overline{\mathcal{D}}_{m, k, j}^{l}(h, s)=\bigcup_{\{x, y, z\} \in A_{k}^{l}(h)}\left\{(w, v, u):\{u, v, w\} \in P_{m, j, s}^{\{x, y, z\}}\right\}, 2 \leqslant h \leqslant \frac{u-1}{2}, 0 \leqslant s \leqslant v-1 .
\end{gathered}
$$

And these $3(u-1) v$ RDTSs are disjoint. We obtain a total of $3(u v-2)$ disjoint $\operatorname{RDTS}(u v)$, a large set. This completes the proof.

\section{New orders}

From Lemma 2.1, 2.2 and Theorem 3.1, we can obtain the following conclusion.

Theorem 4.1 For $v \equiv 0,3,9(\bmod 12)$, if there exists an $\operatorname{LRDTS}(3 v)$, then there exists an $\operatorname{LRDTS}\left(v \cdot \prod_{m_{i} \geqslant 0}\left(2 \cdot 7^{m_{i}}+1\right) \prod_{n_{i} \geqslant 0}\left(2 \cdot 13^{n_{j}}+1\right)\right)$, where $m_{i}$ and $n_{j}$ are non-negative integers.

For example, from Theorem 1.2, for $s \in\{57,93,132,240,255\}$, the existence of LRDTS $(s)$ is unknown. But the existence of $\operatorname{LRDTS}(3 s)$ is known. And from Lemma 2.1, there exists a DTRIQ $(s)$. Thus, from Theorem 4.1, we get the following result.

Theorem 4.2 There exists an $\operatorname{LRDTS}(v)$ for $v=s \cdot \prod_{m_{i} \geqslant 0}\left(2 \cdot 7^{m_{i}}+1\right) \prod_{n_{j} \geqslant 0}\left(2 \cdot 13^{n_{j}}+1\right)$, where $s \in\{57,93,132,240,255\}, m_{i}$ and $n_{j}$ are non-negative integers.

Remark: The smallest order of $v$ (unknown before this paper) obtained from Theorem 4.2 is $1395,1980,3600,3825, \cdots$ in turn. 


\section{References}

[1] J.C. Bermond, A. Germa, D. Sotteau, Resolvable decomposition of $K_{n}^{*}$, J. Combin. Theory Ser. A 26(1979) 179-185.

[2] J. Denes and A.D. Keedwell, Latin Square and their Applications, Academic Press, New York, 1974.

[3] R.H.F. Denniston, Sylvester's problem of the 15 schoolgirls, Discrete Math. 9(1974) 229-238.

[4] R.H.F. Denniston, Double resolvability of some complete 3-design, Manuscripta Math. 12(1974) 105-112.

[5] R.H.F. Denniston, Four doubly resolvable complete 3-design, Ars Combin. 7(1979) 265-272.

[6] R.H.F. Denniston, Further cases of double resolvability, J. Combin. Theory Ser. A 26(1979) 298-303.

[7] L. Ji, J. Lei, Further results on large sets of Kirkman triple systems, Discrete Math. 308(2008) 4643-4652.

[8] Q. Kang, Large sets of resolvable MTS and DTS of order $p^{n}+2$, J. Combin. Des. 4(1996) 301-321.

[9] Q. Kang, Y. Chang, A completion of the spectrum for large sets of disjoint transitive triple systems, J. Combin. Theory Ser. A 60(1992) 287-294.

[10] Q. Kang, J. Lei, On large sets of resolvable and almost resolvable oriented triple systems, J. Combin. Des. 4(1996) 95-104.

[11] Q. Kang, Z. Tian, Large sets of oriented triple systems with resolvability, Discrete Math. 212(2000) 199-221.

[12] Q. Kang, R. Xu, More large sets of resolvable MTS and resolvable DTS with even orders, Acta Math. Appl. Sin. Engl. Ser. 24(2008) 233-252.

[13] Q. Kang, H. Zhao, More large sets of resolvable MTS and resolvable DTS with odd orders, Discrete Math. 308(2008) 886-895.

[14] J. Lei, On the large sets of Kirkman systems with holes, Discrete Math. 254(2002) 259-274.

[15] J. Lei, On the large sets of Kirkman triple systems, Discrete Math. 257(2002) 63-81.

[16] L. Teirlinck, On the maximum number of disjoint Steiner triple systems, Discrete Math. 6(1973) 299-300.

[17] R. Xu, Q. Kang, A construction for $\operatorname{LRMTS}(3 v)$ and $\operatorname{LRDTS}(3 v)$, Journal of Hebei Normal College 2(1997) 8-13. (in Chinese)

[18] L. Yuan, Q. Kang, some infinite families of large sets of Kirkman triple systems, J. Combin. Des. 16(2008) 202-212.

[19] L. Yuan, Q. Kang, Another construction for large sets of Kirkman triple systems, Des. Codes Cryptogr. 48(2008) 35-42. 
[20] S. Zhang, L. Zhu, Transitive resolvable idempotent symmetric quasigroups and large sets of Kirkman triple systems, Discrete Math. 247(2002) 215-223.

[21] S. Zhang, L. Zhu, An improved product construction for large sets of Kirkman triple systems, Discrete Math. 260(2003) 307-313.

[22] J. Zhou, Y. Chang, Tripling construction for large sets of resolvable directed triple systems, Acta Math. Sinica 22(2006) 311-318.

[23] J. Zhou, Y. Chang, Product constructions for large sets of resolvable MTSs and DTSs, Australas. J. Combin. 33(2005) 47-56. 